\title{
A Newly found Bose-Einstein Condensate of G-wave molecules
}

\author{
Atomic Bose-Einstein condensate to molecular Bose-Einstein condensate transition \\ Authors: Zhendong Zhang, Liangchao Chen, Kaixuan Yao, Cheng Chin, \\ arXiv:2006.15297
}

\section{Recommended with a Commentary by Tin-Lun Ho, The Ohio State University}

Recently, Cheng Chin's group at Chicago has reported the realization of a Bose-Einstein condensate (BEC) of G-wave molecules[1]. The molecules are pairs of $\mathrm{Cs}^{133}$ bosons in the orbital $\mathrm{Y}_{42}$ state. They are created by sweeping a BEC of Cs atoms across a G-wave resonance through the variation of an external magnetic field. These molecules are very small $(5.3 \mathrm{~nm})$, much smaller than the inter-molecule spacing (microns). The finding is both surprising and exciting. In the last decade, after the spectacular success of associating fermions into bound pairs through an s-wave Feshbach resonance (hence generating a BEC -BCS crossover in the bulk), there have been continual efforts to use higher angular momentum resonances to create BECs of fermions pairs with non-zero angular momentum, as well as BECs of boson pairs. Until now, such efforts have been unsuccessful due to the serious three-body loss in these systems. The method discovered by the Chicago group to circumvent this loss is therefore a much welcome news.

Connection to condensed matter: This condensate, which we refer to as G-wave BEC, is quite different from superfluid He-3A, even though both are made up of pairs with non-zero angular momentum. Not only are their pairs very different in size, the statistics of their constituent particles are also different. It has been predicted that the transition between atomic and molecular BEC must be first order [2], unlike the BEC-BCS crossover of fermions. This can be seen from their $2 \pi$ vortices. In an atomic BEC, each boson will acquire a phase change of $2 \pi$ around the vortex core, whereas in the molecular BEC, it is the molecule that acquires a $2 \pi$ phase change. This means each boson carries only half of a vortex, and hence cannot be connected smoothly to that in an atomic BEC. With the realization of the G-wave BEC, this prediction can be tested. The G-wave BEC can also shed lights on the longstanding problem of the intrinsic angular momentum of superfluid He-3A. There have been many studies in the last few decades that led to vastly different answers [3]. The issue is about the amount of edge current that mixes with the angular momentum of the Cooper pair. This issue has not been unsettled experimentally as it is difficult to separate different sources of angular momentum. For the G-wave BEC, due to the small size of the molecules, the contribution of the edge current is insignificant. As a result, the intrinsic angular momentum of the pairs can be measured precisely. On the other hand, one can induce edge currents by tightening up the confining trap, or by bringing the system close to 
resonance to increase the pair size. The study of the angular momentum of the G-wave BEC in different physical settings can therefore shed lights on the decades old He-3 problem.

A surprise in atomic physics: Three-body loss is a major destabilizing factor of quantum gases at low temperatures. Two atoms A and B with almost zero energy cannot fall into a deep bound state $(\mathrm{AB})$ due to energy conservation. However, in the presence of a third particle $\mathrm{C}$, the molecule $(\mathrm{AB})$ can form as $\mathrm{C}$ can take away the bound state energy and becomes an energetic particle. As a result, the quantum gas is depleted quickly. This problem is particularly severe near a resonance of the formation of pairs with non-zero angular momentum. Remarkably, by reducing the density of the Cs gas, and by going to a quasi 2D regime, the Chicago group has found a special G-wave resonance where the three-body loss rate is substantially reduced. Moreover, the loss rate reduces as temperature is lowered. As of now, the origin of this reduction is not understood. Yet there is compelling evidence that the molecular gas is a BEC.

Evidence for BEC: Usually, the presence of BEC is identified by the emergence of a sharp peak in the momentum distribution, which can be obtained from the image of an expanding gas after the confining trap is turned off, (i.e. the time-of-flight experiment). However, due to low density of the gas, the Chicago group found it hard to perform the usual time-of-flight measurement in their setting. Instead, they directly measured the equation of state $n(\mu)$ from the density profile $\mathrm{n}(\mathbf{r})$, where $\mu$ is the chemical potential. This method is based on the condensed matter concept of local density approximation, which says the spatial variation of $n(\mathbf{r})$ comes from the change of local chemical potential, i.e. $\mathrm{n}(\mathbf{r})=\mathrm{n}(\mu(\mathbf{r}))$, where $\mu(\mathbf{r})=\mu-\mathrm{V}(\mathbf{r})$, and $\mathrm{V}(\mathbf{r})$ is the external potential. Hence, the density profile in a confining trap immediately gives the equation of state over a range of chemical potentials. Since the equation of state of a quantum gas takes on very different forms depending on whether it is in the normal or the condensed phase, and whether it is an atomic BEC or a molecular BEC, the Chicago group was able to identify the BEC phase of the G-wave molecules from their density profiles.

Outlook: Currently, the experiments on G-wave BEC are performed far away from resonance where the molecules are very small and are weakly interacting. This naturally raises the question about the physics near resonance, where the molecules are bigger and are strongly interacting. It is conceivable that new types of universal behavior may emerge, that is different from the known universality of strongly interacting Fermi gas. At the same time, the G-wave BEC provides opportunities to study some fundamental features of condensed matter that are be difficult to explore with conventional methods. Take the half vortex as an example. A likely 2-body wavefunction function for the half vortex that satisfies Bose statistics is $\Psi\left(\mathbf{r}_{1}, \mathbf{r}_{2}\right)=e^{i \theta\left(\mathbf{r}_{1}, \mathbf{r}_{2}\right)} f\left(\mathbf{r}_{1}\right.$ $\left.\mathbf{r}_{2}\right)$, where

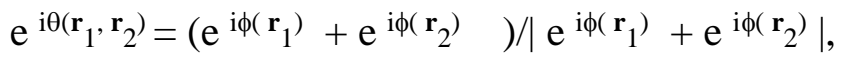

$\phi(\mathbf{r})$ is the azimuthal angle of $\mathbf{r}$, and $\mathrm{f}(\mathbf{r})$ is a radial function of the tightly bound molecule. Such a function will have signatures in the interference pattern of two G-wave condensates that can be revealed in time-of-flight experiments. The realization of the G-wave BEC suggests other molecular BECs with non-zero angular momentum may also be created in the future. Should this happened, the study of molecular BEC will flourish further as there will be new physics of the mixtures these condensates. 


\section{References}

[1] Zhendong Zhang, Liangchao Chen, Kaixuan Yao, Cheng Chin, Atomic Bose-Einstein condensate to molecular Bose-Einstein condensate transition, arXiv:2006.15297

[2] M.W.J. Romans, R.A. Duine, S. Sachdev, and H. T. C. Stoof, Quantum phase transition in atomic Bose gas with a Feshbach resonance. Physical Review Letters 93, 020405 (2004); L. Radzihovsky, J. Park, and P.B. Weichman, Superfluid transition in bosonic atom-molecule mixture near a Feshbach resonance. Physical Review Letters 92, 160402 (2004).

[3] P. W. Anderson and P. Morel, Generalized Bardeen-Cooper-Schrieffer States of the Proposed Low-Temperature Phase of Liquid He ${ }^{3}$, Physical Review 123, 1911, (1961). M. Ishikawa, Orbital Angular Momentum of Anisotropic Superfluid, Progress of Theoretical Physics 57, 1836, (1977). M.C. Cross, A Generalized Ginzburg-Landau Approach to the Superfluidity of Helium 3, Journal of Low Temperature Physics 21, 525, (1975). Michael Stone and Rahul Roy, Edge modes, edge currents and gauge invariance in $p_{x}+i p_{y}$ superfluids and superconductors, Physical Review B 69, 184511, (2004). Y. Tsutsumi, T, Mizushima, M. Ichioka, K. Machida, On Intrinsic Angular Momentum due to Edge Mass Current for Superfluid ${ }^{3}$ He A-Phase, Journal of Physics: Conference Series 400012076 (2012) 